\title{
„Und ja, ich weiß, dass wir uns nicht kennen, aber ..... Epistolare Distanzspiele
}

\section{Gespräche unter Abwesenden}

Am Anfang der rhetorischen Brieftheorie steht die Distanz: Im ersten Jahrhundert nennt Artemon im Vorwort seiner Aristoteles-Briefedition „den Brief die eine Hälfte eines Dialoges, den Briefwechsel ein Gespräch unter Abwesenden. “1 Die Edition selbst ist zwar nicht erhalten geblieben, dennoch haben wir ihr einen so langlebigen wie wirkmächtigen Epistolartopos zu verdanken, der seitdem von Jahrhundert zu Jahrhundert weitergereicht worden ist. ${ }^{2}$ Als ,Wechselrede unter Freunden in Abwesenheit' (amicorum conloquia absentium) versteht denn auch die für die humanistische Briefkultur zentrale Autorität Cicero den Brief. ${ }^{3}$ Und noch Christian Fürchtegott Gellert, dessen Natürlichkeitsemphase Anlass zu so manchem interpretatorischen Missverständnis gegeben hat, versteht den Brief in bester rhetorischer ${ }^{4}$ Tradition als eine - wenn auch „freye“ - „Nachahmung des guten Gesprächs. "5 Gellert beruft sich namentlich auf Seneca, der in einem Brief an Lucilius erklärt hatte: „Wie mein Stil beschaffen wäre, wenn wir zusammensäßen oder spazierengingen, nämlich ungezwungen und salopp, so sollen meine Briefe

1 Vgl. Ueding 2016, 21.

2 Vgl. Ueding 2016, 21; Fürholzer et al. 2017, 7.

3 Vgl. Ueding 2016, 22.

4 Gert Ueding macht auf die tiefe Verankerung Gellerts in der rhetorischen Tradition aufmerksam: „Von europäischer Briefkultur sprechen, das heißt von Rhetorik sprechen, auch wenn das Bewusstsein dieser Zuständigkeit durch eine oberflächliche Lektüre von Gellerts epistolographischer Reform verloren gegangen ist. Dessen Distanzierung von Rhetorik bezog sich auf den Stylus Curiae, der seit dem Mittelalter die Briefstellerei dominierte. Gellert selber befindet sich aber ganz in rhetorischer Überlieferung, in seinem Kampf gegen die Curialstil-Rhetorik bediente er sich der klassischen Quellen; er zitiert Cicero und Quintilian und bringt allenfalls mit seiner Betonung des Anmutig-Freundlichen einen eigenen Akzent in die Gattungsgeschichte. Im übrigen [sic!] hält er sich sowohl was das prinzipielle Verständnis wie auch die einzelnen Empfehlungen und die Methode der Verbesserung der deutschen Briefkultur betrifft an seine antiken Quellen. “ (Ueding 2016, 21.)

5 Teilzitate: Gellert 1751, 3.

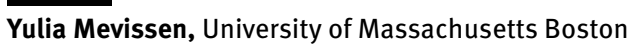

○ Open Access. (C) 2021 Yulia Mevissen, publiziert von De Gruyter (c)) BY-NC-ND Dieses Werk ist lizenziert unter der Creative Commons Attribution-Non-Commercial-NoDerivatives 4.0 International Lizenz.

https://doi.org/10.1515/9783110712568-017 
sein, wünsche ich, die nichts Gesuchtes enthalten und Gekünsteltes.“6 [„,Qualis sermo meus esset, si vna sederemus, aut ambularemus, illaboratus \& facilis: tales esse epistolas meas volo, quae nihil habeant accersitum nec fictum. "]

Beide Teile von Artemons Bestimmung des Briefes als ,Gespräch unter Abwesenden' sind von eminenter Relevanz: Die ,Abwesenheit' von Sender und Empfänger in schriftbasierter Kommunikation (,Gespräch unter Abwesenden') und der Gesprächscharakter (,Gespräch unter Abwesenden'), der ebendiese physische ,Abwesenheit' konzeptionell-imaginär gleich wieder aufzuheben (wenn auch nicht restlos zu eliminieren) vermag. Diese in der als paradoxal empfundenen Losungsformel vom ,Gespräch unter Abwesenden' inbegriffene Aufhebungsbewegung als Hinwendung zum (Gegen-)Pol der Mündlichkeit lesend (was auch immer im Einzelnen darunter zu verstehen sein mag), hat man in der Folge mal mehr, mal weniger naive Gleichheitszeichen zwischen epistolarem ,Gespräch', (konzeptioneller) Mündlichkeit und Nähe gezogen. Insofern sich die frühe Briefforschung in einer sagenhaften Korpusverengung auf den Freundschaftsbrief sentimentaler Couleur bzw. den empfindsamen Briefroman konzentrierte und diesen als prototypisch für das ,Wesen der Briefgattung schlechthin erklärte, ${ }^{8}$ universalisierte und essentialisierte sie eine sehr bestimmte, historisch datierbare Vorstellung vom ,Brief-Gespräch` in seiner Spielform als freundschaftlichem ,Gespräch - und auch die irgendwie intuitiv eingängige Gleichung mit dazu, wonach Dialogizität synonym mit Mündlichkeit zu denken sei und (konzeptionelle) Mündlichkeit wiederum als gleichbedeutend mit Nähe. Aber was eigentlich heißt hier ,Mündlichkeit ‘ und wie genau ist ,Nähe` zu operationalisieren? ${ }^{9} \mathrm{Ganz}$ so einfach, wie es dem Wortsinne nach scheinen mag, ist es nämlich gerade um die Mündlichkeitsorientierung nicht bestellt.

Man nehme hier nur einmal das Beispiel Gellert. Bekanntlich aktualisiert die so gern erforschte Briefkultur der Empfindsamkeit den antiken Topos vom epistolaren ,Gespräch unter Abwesenden', den sie in das Postulat der ,Natürlichkeit“ ummünzt. Nicht einmal Gellert aber, theoretischer Gewährsmann, wann immer es

6 Hier im Wortlaut der von Manfred Rosenbach besorgten Übersetzung wiedergegeben: Seneca 1987, 85, Brief 75.2.

7 Gellert 1751, 3, Anm.

8 Vgl. Nickisch 1991, 2 u. ö. Diese Beobachtung liegt auch Siegerts Kritik an einer ,anthropozentrischen Brieftheorie“ zugrunde (vgl. Siegert 1993, bes. 20-21 u. 82).

9 Die Konzeptualisierung von Mündlichkeit als „Sprache der Nähe“, von Schriftlichkeit hingegen als „Sprache der Distanz“, stammt aus der Textlinguistik und ist entsprechend vor dem Hintergrund eines gänzlich anderen Frageinteresses entwickelt worden; medientheoretische Aspekte oder Epistolargeschichte lagen dabei außerhalb des gesetzten analytischen Fokus. Grundlegend: Koch et al. 1985. Zur Rezeptionsgeschichte dieser Konzeptualisierung vgl. Oesterreicher et al. 2016. 
um das Phantasma einer mündlichkeitsorientierten, ,natürlichen‘ Immediatkommunikation geht, ist das tatsächliche Gespräch, so wie es im Alltag geführt wird, wirklich geheuer: Es sei „gar zu natürlich[]“, ${ }^{10}$ erklärt Gellert abfällig. Nicht um die Kopie eines mündlichen Gespräches, von der Sorte, wie es realiter alltagsweltlich geführt wird oder geführt werden könnte, geht es Gellert, sondern um einen sorgsam erzeugten Natürlichkeitseffekt, ${ }^{11}$ für den das ,Gespräch' nur als griffigeinprägsame Chiffre einsteht: „Zuweilen kostet eben das Leichte, das Natürliche“, führt Gellert aus, „die meiste Mühe, und gefällt doch dem Leser am Ende aus dem Grunde, weil es keine Mühe gekostet zu haben scheint. Man hat alle Arbeit, alle Kunst versteckt." ${ }^{12}$ Die Rhetorik kennt das als dissimulatio artis oder auch artem celare-Prinzip. Mit tatsächlicher Mündlichkeit hat dies reichlich wenig zu tun.

\section{Epistolare Distanzen}

Aber zurück zur epistolaren Größe ,Distanz‘. Wer es mit Briefen zu tun hat, sieht sich mit ,Distanzen“ höchst unterschiedlicher Art konfrontiert. Da wäre zunächst einmal der seit Artemon bekannte, wenn auch keineswegs banale, Umstand, dass Briefe als temporal und spatial ,zerdehnte ${ }^{613}$ Rede eine Distanzkommunikation zwischen physisch ,Abwesenden' ermöglichen. ${ }^{14}$ Nicht nur aber raumzeitliche Distanzen im ganz literalen Sinne gibt es brieflich zu überwinden, sondern auch eher figurative zwischenmenschliche ,Distanzen' zwischen Sender und Empfänger, deren Regulation und Modulation Briefe als ,symbolische Distanzäquivalente“ übernehmen können, wie es Robert Vellusig in Rekurs auf Norbert Bischof griffig formuliert hat. ${ }^{15}$ Während raumzeitliche Distanzen qua Zollstock und Uhr sich ohne Weiteres objektiv feststellen lassen, sind zwischenmenschliche ,Distanzen“

10 Gellert 1751, 15.

11 Natürlichkeit ist, wie Robert Vellusig schreibt, ,keine genetische, sondern eine qualitative Kategorie: sie bestimmt sich nicht über die Entstehungsbedingungen der Texte“ (Vellusig 2011, 165).

12 Gellert 1751, 14.

13 Konrad Ehlich prägt den Begriff der „zerdehnten Sprechsituation“, während Peter Bürgel die Wendung vom „brieftypischen Phasenverzug “ in die literaturwissenschaftliche Sprache einführt (Ehlich 1983, 32; Bürgel 1976, 288).

14 In spezifischer Weise ist der Begriff der Anwesenheit (in Opposition zur Abwesenheit) in der Geschichtswissenschaft fruchtbar gemacht worden, um die für die Frühe Neuzeit charakteristische gesellschaftliche Interaktion konzeptuell zu fassen. Rudolf Schlögl hat die Frühe Neuzeit prominent als „Vergesellschaftung unter Anwesenden“ beschrieben: Schlögl 2014.

15 Zur symbolischen Distanzregulierung durch Briefe in Rekurs auf Norbert Bischof vgl. Vellusig 2018, 64-72; Vellusig 2011, 166. 
psychische Größen, die sich bei Sender und Empfänger weder gleichen müssen ${ }^{16}$ noch sich durch eine simple Formel in ein Verhältnis zur raumzeitlichen Distanz als anderer Achse eines etwaigen Koordinatensystems bringen ließen (physische Distanz kann psychisch-emotive Distanz erzeugen bzw. steigern - oder eben im Gegenteil verringern, ja, vielleicht sogar nahezu zum Verschwinden bringen). Es sind qualitativ unterschiedliche Kategorien, die miteinander korrelieren können, aber nicht müssen.

In jedem Fall aber ist ,Distanz“ - selbst wenn sie sich als graduierbare Größe versteht - keine neutrale Beschreibungskategorie, sondern eine Defizitdiagnose. ,Nähe“ und ,Distanz' sind keine grundsätzlich gleichwertigen Extrempunkte einer Skala, und Distanzen gilt es schon redensartlich zu ,überwinden‘. An diesem konnotativen Ballast ändert sich prinzipiell auch nichts, wenn die Linguisten Peter Koch und Wulf Oesterreicher, denen die Wissenschaftssprache die Metaphern der „Sprache der Nähe“ bzw. „Sprache der Distanz“ verdankt, darauf insistieren, dass mit dem Terminus Distanz ,in keinem Fall eine abwertende Nuance verbunden werden [darf]. “17

Die briefliche Kommunikation hat - gemessen am normativ der face to faceInteraktion zugesprochenen Primat - zunächst einmal sekundären, kompensatorischen Charakter. Unmissverständlich ausgedrückt wird dieser epistemologische Umstand beispielsweise in Sophie von La Roches empfindsamem Briefroman $\mathrm{Ge}$ schichte des Fräuleins von Sternheim (1771): „Da mich das schlimme Wetter und eine kleine Unpässlichkeit abhalten, meiner gnädigen Mama selbst aufzuwarten, so will ich doch meinem Herzen das edle Vergnügen nicht versagen, mich schriftlich mit Ihnen zu unterhalten. "18 Hier wird zum Stift gegriffen, weil äußere Umstände (das Wetter, eine kleine Unpässlichkeit) dazu schlechterdings nötigen. Der Stift ist Kommunikationsmittel zweiter Wahl. Die schriftliche ,Unterhaltung - aufgerufen wird auch hier wieder einmal der obligate Topos vom Brief als ,Gespräch unter Abwesenden' (sermon absentis ad absentem) - steht für die nicht zustande gekommene mündliche Unterhaltung ein; die Distanzkommunikation ist konzeptionell Substitut der Nahkommunikation.

Ganz so einfach ist es dann aber doch nicht. Erstens nämlich ist der - dem eigenen Epochenparadigma verpflichtete - Ratschlag des „Schreibe wie Du redest“ ${ }^{19}$

16 „Als psychologische Kategorie ist Distanz ein asymmetrisches Phänomen: Die Distanz, die A zu B einnimmt, ist mit der Distanz von B zu A nicht identisch. A kann sich B nahe fühlen, während B A ignoriert oder sich ihm gegenüber distanziert verhält.“ (Vellusig 2018, 64).

17 Koch et al. 1985, 21, Anm. 17.

18 [La Roche] 1771, Bd. 1, 38.

19 „Schreibe wie Du redest, so schreibst Du schön“, hatte Gotthold Ephraim Lessing der älteren Schwester Dorothea Salome Lessing im Brief vom 30. Dezember 1743 geraten. (Lessing 1967, 4). 
wie gesagt, nicht zu wörtlich zu nehmen: „Wer Briefe schön schreiben will“, erhellt Christoph Fürchtegott Gellert normativ, ${ }^{20}$ „muß nicht so wohl schreiben, wie ein jeder im gemeinen Leben reden [...] würde“,,21 und warnt in moralethischer und stilistischer Hinsicht, wie zuvor erwähnt, vor einer „gar zu natürlichen Schreibart“.22 Zweitens eignet zwar ausgerechnet dem Distanzmedium Brief als ästhetischer Ausdrucksform eigener Dignität ein affektives Maximierungspotential, mit Husserl gesprochen: die Fähigkeit zur Appräsentation ${ }^{23}$ fremden Bewusstseins und - gerade aufgrund der Abwesenheit des Anderen - eine zumindest bis zur nächsten persönlichen Begegnung vom leidigen Realitätscheck suspendierte Gefügigkeit zu emotiven Besetzungen, Projektionen und Fetischisierungen aller Art an. ${ }^{24}$

Gerade in der Schrift aber ist die Distanz immer schon prinzipiell als lauernde Gefahr angelegt. Immerhin sind schriftliche Zeichen symbolische und also artifizielle Zeichen. Wenn das natürlichkeitsversessene - in der literaturwissenschaftlichen Briefforschung traditionell gern behandelte - achtzehnte Jahrhundert dennoch eine epistolare Immediatkommunikation, als ,Herzenskommunikation“ zumal, imaginiert, wird dieser semiotische Abgrund ausgeblendet, der andernorts in der Epoche eifrigst diskutiert wird.

Wer heute - im einundzwanzigsten Jahrhundert - Briefe oder gar Briefromane sich zu schreiben vornimmt, hat es außerdem noch mit einer historischen Distanz zu tun, die als Distanz ästhetisch aufgegriffen und umspielt werden kann. So zollt etwa Feridun Zaimoglus Protagonist Hakan der Briefromantradition negativen, ironisch-parodistischen Tribut, wenn er (in einem Briefroman, wo sonst?) schreibt: „Hör auf mit der Goethe-Nummer, pfeif drauf und lass einfach die Wolken ziehn, derweil du durch die Sonnengläser faul und ölig blinzelst. “25 Während das populäre Sachbuch genüsslich die digitale Moderne und die ,alte‘ Briefkultur als unversöhnliches Gegensatzpaar darstellt und gegeneinander ausspielt, boomt die Briefforschung und machen erst die Digital Humanities umfangreiche Briefkorpora auf ganz neue Weise zugänglich. ${ }^{26}$ Auch die Gattung Briefroman erlebt gegenwärtig einen Aufschwung. ${ }^{27}$ Und das alles allen kulturpessimistischen Unkenrufen zum

20 Zur Normativität von Empfindsamkeit und Natürlichkeit vgl. Wegmann 1988.

21 Gellert 1751, 10

22 Gellert 1751, 15.

23 Zum Begriff der Appräsentation bei Edmund Husserl und seiner Weiterentwicklung bei Alfred Schütz vgl. überblicksartig Stuck 2013.

24 Vgl. zu emotiven und libidinösen Besetzungsvorgängen Beebee 2006, bes. 48-50.

25 Zaimoglu 2002, 18.

26 Zu den Verdiensten digitaler Briefeditionen vgl. Strobel 2018.

27 Es scheint sich dabei um ein gesamteuropäisches Phänomen zu handeln, wie schon Thomas

O. Beebee vermerkt: Beebee 2006, 199-205. 
Trotz; mindestens seit den 60er Jahren schon wird regelmäßig ein (vermeintliches) „Ende der Briefzeit“ betrauert. 28

\section{Tilman Rammstedt und die literarische Vermessung elektronischer Distanzen}

Der Umgang mit der Größe ,Distanz‘ ist nicht zuletzt das, was Briefe von ihren heute allseits präsenten elektronischen Counterparts trennt. Auch E-Mails, SMS, WhatsApp-Nachrichten und dergleichen sind - mit Artemon gesprochen - ,Gespräche unter Abwesenden', sind spatial ,zerdehnte' Kommunikation. Sie sind jedoch nicht mehr in dem gleichen Maße temporal ,zerdehnte‘ Kommunikation, wie Gert Ueding argumentiert hat:

Die elektronischen Medien haben das Briefgespräch verändert, nicht weil der Bildschirm an die Stelle des Papiers getreten ist, sondern weil der zeitliche Abstand zwischen Brief und Antwortbrief extrem eingezogen wird und es nur an Präsenz und Willen des Adressaten liegt, ihn im Moment des Absendens zu lesen und sogleich zu beantworten. ${ }^{29}$

Dass die digitale Medienrevolution nicht ohne literarische Folgen blieb, nimmt eigentlich nicht groß Wunder. Im empraktischen Vollzug reflektiert die inzwischen gar nicht mehr so neue Subgattung der E-Mail- oder auch Facebook-Romane, was es heißt, ,Gespräche unter Abwesenden` im digitalen Zeitalter zu schreiben. Um hier nur ein paar wenige Beispiele zu nennen: Der in 28 Sprachen übersetzte und seit dem Herbst 2019 nun auch fürs Kino verfilmte E-Mail-Roman-Bestseller Gut gegen Nordwind (2006) des Österreichers Daniel Glattauer zeichnet die virtuelle Annäherung zweier Unbekannter durch den Austausch von E-Mails nach; der Roman zeigt die durch elektronische Kommunikationsmedien ermöglichte TempoBeschleunigung, wenn er den teils sehr kurzen E-Mail-Mitteilungen seiner Figuren Angaben voranstellt wie „Eine Woche später“, „Drei Minuten später“ oder nur „30 Sekunden später“. ${ }^{30}$ Auch Senthuran Varatharajahs im Jahre 2016 im S. Fischer Verlag veröffentlichter, mehrfach ausgezeichneter Debütroman Vor der Zunahme der Zeichen, der ebenfalls zwei Fremde eine ,Unterhaltung führen lässt, via Facebook allerdings, operiert mit der Variable ,Zeit'. Varatharajah versieht die im Roman

28 Vgl. Hess-Lüttich 1997, 225-226.

29 Ueding 2016, 33.

30 Alle Teilzitate: Glattauer 2008, 28. 
abgedruckten Nachrichten mit Uhrzeiten und - zur Markierung vom Smartphone gesendeter Mitteilungen - mit Telefonsymbolen am Rand. ${ }^{31}$

Gerade aber diese Angaben sind es, die den Hiatus zwischen dem Roman und einem ,realen' Computer-Interface letztlich doch nur perzeptibel machen, die Aufmerksamkeit auf das Medium selbst richten und einen naiven SimulacrumCharakter verunmöglichen. Informationen wie „30 Sekunden später“ finden sich in einem E-Mail-Roman, nicht jedoch in ,wirklichen' E-Mail-Accounts; und anders als der ,echte‘ Facebook Messenger verzichtet der Facebook-Roman nicht nur auf Datumsangaben, sondern auch auf die Mimesis der graphischen Qualitäten des Messengers. Das aber macht alles überhaupt nichts. Simuliert wird hier schließlich die verkürzte temporale ,Zerdehnung“ der Kommunikation, nicht das Medium selbst.

Die gleiche Grund-Konstellation (namentlich: die Annäherung an einen Unbekannten qua körperloser, elektronischer Kommunikationsmedien; Verringerung der psychischen Distanz trotz oder gerade aufgrund der Beibehaltung räumlicher Distanz in einem digitalen Nachrichtenaustausch) liegt auch dem im Jahre $2012 \mathrm{im}$ Kölner DuMont Verlag erschienenen, metafiktionalen E-Mail-Roman von Tilman Rammstedt mit dem Titel Die Abenteuer meines ehemaligen Bankberaters zugrunde. Anders als in Glattauers E-Mail-Roman oder Varatharajahs Facebook-Roman wird hier auf eine Authentizitätssuggestion durch vorangestellte Zeit-Angaben verzichtet. Vergeblich auch sucht man nach Datumsangaben, der E-Mail-Adresse von Sender und Empfänger oder etwa der für E-Mail-Kommunikation charakteristischen Betreff-Zeile.

Darin allerdings nun ist Rammstedts E-Mail-Roman seinem kommunikationstechnologischen Vorgänger, dem Briefroman, gar nicht einmal so unähnlich, der zur Generierung seines ,epistolaren' Charakters keineswegs auf die getreue Mimesis der materialen (oder auch nur formalen) Eigenschaften eines Briefes angewiesen ist. Literarische Briefe, wie sie etwa Sophie von La Roches Geschichte des Fräuleins von Sternheim präsentiert, kommen nicht nur ohne Kuvert, Adressangabe und Datumszeile an die Leserschaft, sondern haben noch nicht einmal notwendigerweise eine Anrede- und eine Grußformel, die den Briefinhalt rahmen würde. (Soviel zur durch die Forschungslandschaft geisternden Illusionierungsthese, dass in der Gattung Briefroman dadurch, dass gewissermaßen die Dokumente ,selbst“ in ihrer Materialität vorlägen, Fakt und Fiktion bis zur Ununterscheidbarkeit zur Oszillation kämen.)

Tilman Rammstedts absurd-komischer, monologischer E-Mail-Roman Die Abenteuer meines ehemaligen Bankberaters baut sein ganzes literarisches Spiel

31 Varatharajah 2016. 
darauf auf, dass ein Autor, der den Namen Tilman Rammstedt trägt, dem USamerikanischen Actionfilmstar Bruce Willis eine E-Mail schickt und, da diese unbeantwortet bleibt, weitere Nachrichten nachfolgen lässt, die ebenso ohne Antwort bleiben. Tatsächlich erstreckt sich die Schweigsamkeit des Filmstars noch bis auf das kongeniale Cover, das aus einer weiteren - wieder: unbeantworteten E-Mail der Autor-Persona an die Bruce-Willis-Figur besteht.

In dieser E-Mail bittet die Autor-Persona nicht nur in metaleptischer Verlängerung des im Roman entfalteten metafiktionalen Spiels um ein Zitat für den Schutzumschlag des Buches, sondern legt die erbetenen Zitate der Bruce-WillisFigur als fingierte Rede auch sogleich in den Mund (die Rhetorik kennt eine solche Strategie als Figur der sermocinatio). Wer nur einen flüchtigen Blick auf das Cover wirft, sieht tatsächlich zunächst einmal nur die durch Fettdruck abgesetzten ,Zitate‘. Faltet man den Schutzumschlag der Hardcover-Ausgabe auf (der Taschenbuchausgabe entgeht diese paratextuelle Pointe), wird der Buchtitel Die Abenteuer meines ehemaligen Bankberaters nicht nur relativiert durch den konjunktivischen Zusatz wie ich sie gerne erlebt hätte zumindest am Anfang und ein wenig noch am Ende, sondern die auf dem Buchumschlag abgebildete Silhouette von Bruce Willis durch die Silhouette Tilman Rammstedts ersetzt. In ein Wechselbild übersetzt wird hier gewissermaßen das ganze Darstellungsprinzip des Romans.

Man kann, wenn man so will, Rammstedts E-Mail-Roman als monologischeinseitigen Versuch lesen, die psychische Distanz zum Empfänger zu reduzieren. Da dies durch wiederholtes Anschreiben und die beharrliche Repetition der Bitte, doch einmal zurückzuschreiben, nur bedingt gelingt, da ja doch nun einmal nie eine Antwort zurückkommt, bleibt als letzter Ausweg nur noch das literarische Mittel der sermocinatio, d. h. der fingierten Rede, der unterstellten Handlung. Diese - im Übrigen erfolgreiche - Strategie wird durch die dem Medium inhärente raumzeitliche Distanz durchaus begünstigt.

Am Beginn des Romans (der neben E-Mails auch sentenzhafte Aussprüche der titelgebenden Figur des ehemaligen Bankberaters enthält, die alternierend abgedruckt werden) steht der erste Versuch der Kontaktaufnahme der Autor-Persona mit Bruce Willis:

Sehr geehrter Herr Willis, geht es Ihnen gut? Mit freundlichen Grüßen, Tilman Rammstedt ${ }^{32}$

32 Rammstedt 2012, 5. 
Insofern die Frage how are you? im amerikanischen Englisch nicht viel mehr als eine semantisch entleerte Grußformel darstellt, mit der man vom Supermarktverkäufer bis zum besten Freund jedermann und jedefrau begrüßt, handelt es sich hier um eine propositional maximal arme E-Mail, deren einziger Satz im Grunde nur eine redundante Doppelung der Anrede ist. ${ }^{33}$ Weiter auf die Spitze getrieben wird dies nur noch von der E-Mail:

Sehr geehrter Herr Willis,

Ihr

Tilman Rammstedt ${ }^{34}$

Den erwünschten Effekt, nämlich eine Reaktion des Anderen zu erzwingen und dadurch einen elektronischen Briefwechsel herzustellen, zeitigen diese E-Mails nicht. Weder eine verbale Antwort in Form einer E-Mail kommt zurück noch wird dem Vorschlag der Autor-Persona gefolgt, sich per Handy oder via Skype zu melden, ${ }^{35}$ „ein aussagekräftiges Foto [zu] schicken“36 oder doch wenigstens nur ein einziges Wort zu mailen, „ein ,Ja' zum Beispiel, ein ,Bald‘, meinetwegen auch ein ,Hm‘. Das wäre ein Anfang. Auf ,Hm` könnte man aufbauen. “37 Worum es hier geht, ist nicht propositionaler Gehalt und konversationeller Tiefsinn, sondern der phatische Aufbau einer Beziehungsebene (und hierfür wäre tatsächlich schon ein bloßes ,Hm' geeignet in seiner semantischen Unterspezifiziertheit als Gesprächspartikel). Doch nicht einmal eines ,Hms“ würde es für den Aufbau einer Beziehungsebene bedürfen: „Schreiben Sie mir gar nichts, antworten Sie einfach mit einer leeren Mail, auch das ist ein erster Schritt.“38

Die von Rammstedt gewählte Anrede- und Grußformel ist förmlich (und bleibt es auch den gesamten Roman über); es wird gesiezt. In seiner zweiten E-Mail an Willis konzediert die schreibende Autor-Persona den Grund für die ausbleibende Antwort recht klarsichtig: „[...] ja, ich weiß, dass wir uns nicht kennen [...].“39 Doch schon ab der vierten E-Mail hat sich dies geändert; nun heißt es schon, dass „wir uns [jetzt] ein wenig kennen“.40 Und tatsächlich ,kennt“ die Autor-Persona

33 Dass dies nicht so gemeint war, bedarf erst des klärenden Nachsatzes in der Folge-E-Mail: „Sehr geehrter Herr Willis, die Frage in meiner gestrigen Mail war übrigens nicht als höfliche Floskel gemeint.“ (Rammstedt 2012, 5).

34 Rammstedt 2012, 56.

35 Vgl. Rammstedt 2012, 9.

36 Rammstedt 2012, 11.

37 Rammstedt 2012, 11.

38 Rammstedt 2012, 19.

39 Rammstedt 2012, 5.

40 Rammstedt 2012, 8. 
den angeschriebenen Actionfilmstar nicht nur aus den Medien, sondern nutzt ihn - vor dem Hintergrund der eigenen, nur in Andeutungen und oftmals nur eher beiläufig in Parenthese bezeichneten Lebensprobleme - als Projektionsfigur, von der er sich ausmalt, was diese gerade „wahrscheinlich“, ${ }^{41}$ „vielleicht“42 oder auch „bestimmt“ 43 tut, denkt oder fühlt. Schreibend verringert sich, von E-Mail zu E-Mail, in rasantem Tempo die psychische Distanz, was in komischen Kontrast zur förmlich bleibenden Anrede- und Grußformel rückt, die mit dem umgangssprachlich-vertrauten Ton der Korrespondenz kontrastiert. Gerade weil die raumzeitliche Distanz nicht in der realweltlichen, persönlichen Begegnung aufgehoben wird, gerade weil der Andere den Schriftverkehr außerdem nicht durch eine Antwortmail zum Schriftwechsel werden lässt, lässt sich unwidersprochen beidseitige psychische Nähe behaupten, wo sie lediglich einseitig ist. So unterstellt schließlich die Autor-Persona Bruce Willis, dass er ja „gar nicht wolle[]“ ,44

dass ich Ihnen nicht mehr schreibe. Sie ertappen sich doch sogar ab und zu dabei, enttäuscht zu sein, wenn eine neu eingehende Mail nicht von mir ist. Wir sind für einander doch längst Teil unseres Lebens geworden. Wir können uns doch gar nicht mehr genau erinnern, wie es ohne den anderen war.

Ist es nicht so, Herr Willis? Ist es nicht genau so? ${ }^{45}$

Nicht nur wird hier kurzerhand ein „wir“ behauptet, sondern durch rhetorische Fragen, nicht zuletzt aber durch die Modalpartikel „doch“, sprachlich-performativ eine Nähe konstruiert. Schließlich suggeriert die - im Übrigen eine Affinität zur gesprochenen Sprache aufweisende - Modalpartikel „doch“ wissensökonomisch eine Referenz auf Selbstverständliches und Bekanntes, suggeriert einen Bezug auf das Vorwissen des Anderen bzw. mehr noch: auf ein gemeinsames Wissen. Durch eine extensive Verwendung von Modalpartikeln in dieser Funktion wird Nähe sprachlich-rhetorisch hergestellt. Es sind gerade die kleinen, unauffälligen Synsemantika, die zu Unrecht als inhaltslose Füllwörter verschrienen Partikel, die einen sehr großen Beitrag zur sprachlichen Produktion von Nähe leisten, insofern sie ein gemeinsames Zeigfeld ${ }^{46}$ aufbauen.

Aber worum eigentlich geht es in dem Roman? Der Plot des E-Mail-Romans geht folgendermaßen: ,Tilman Rammstedt‘ bietet ,Bruce Willis‘ eine Rolle in seinem

41 Rammstedt 2012, 8 u. ö.

42 Rammstedt 2012, 9 u. ö.

43 Rammstedt 2012, 9 u. ö.

44 Rammstedt 2012, 27.

45 Rammstedt 2012, 27.

46 Zur Fruchtbarkeit von Karl Bühlers „Zeigfeld“-Konzept vgl. Ehlich 2009, 69-70, der selbst den Terminus des „Verweisraums“ nutzt. 
neuen Roman an, dessen Titel in schönster metafiktionaler Manier dem ,realen` Titel des Buches gleicht. Als eine Art „Body-Double“47 soll Bruce Willis für den Protagonisten des im Entstehen begriffenen Romans, den Bankberater, einspringen und ihn aus einer „brenzligen Situation“48 erretten, um „ein glückliches Ende“49 herbeizuführen. Der (ehemalige) Bankberater nämlich hat seine eigene Bank überfallen und steht jetzt „mitten im Überfall, mitten in der brenzligen Situation“ ${ }^{50}$ und „weiß nicht weiter“. ${ }^{51}$ Aus dieser in medias res-Situation nun wird eine zeitliche Dringlichkeit generiert, „genau jetzt“ $z u$ agieren und das heißt zuvorderst: endlich der längst überfälligen Briefschuld nachzukommen. „Sehr geehrter Herr Willis“, schreibt die Autor-Persona,

es wäre auf jeden Fall der richtige Moment, mir endlich zu schreiben. Genau jetzt.

Bis gleich,

Ihr

Tilman Rammstedt ${ }^{52}$

Und da eine Antwort weiterhin ausbleibt:

Herr Willis, genau jetzt.

$\mathrm{TR}^{53}$

Immer wieder wird ein zeitlicher „Aufschub“54 gewährt. Literarisch ausgespielt werden die Möglichkeiten des Kommunikationsmediums E-Mail, die temporale Distanz zwischen Absenden und Ankommen einer Nachricht - und folglich potentiell zwischen Absenden und Lesen und Reagieren in Form einer eigenen Nachricht auf ein Minimum einzudampfen. Gerade das aber macht die Nicht-Antwort des Anderen umso schwerer wiegend. Verweigert wird sich hier nicht nur der - freilich an Cyberstalking grenzenden - eingehenden Nachrichtenflut, sondern auch dem Erreichbarkeitsgebot des Mediums selbst, das heute in seiner Verschwisterung mit E-Mail-Abruf-Apps auf dem Smartphone tatsächlich der Utopie der Synchronizität erstaunlich nahekommt und die Grenzen zu anderen ,mobilen', auf ständige Er-

47 Eine prompte Zurückweisung dieser Rolle wird Bruce Willis freilich sogleich in den Mund gelegt. Vgl. Rammstedt 2012, 27.

48 Rammstedt 2012, 30 u. ö.

49 Rammstedt 2012, 24 u. ö.

50 Rammstedt 2012, 30.

51 Rammstedt 2012, 34.

52 Rammstedt 2012, 39.

53 Rammstedt 2012, 39.

54 Rammstedt 2012, 41. 
reichbarkeit gepolten Kommunikationsformen wie etwa der SMS verschwimmen lässt. Nur zu bezeichnend ist, wenn die Wissenschaftsmetaphorik der Linguistik inzwischen das „Konzept der Online-Zeitlichkeit“ hervorgebracht hat, das - insofern es für eine „raum-zeitliche[] Synchronizität“ der Kommunikation einsteht wiederum „als relevantes Merkmal für gesprochene Sprache“ diskutiert wird. ${ }^{55}$

Aber zurück zu Rammstedts Roman. Zwar fruchtet die Poetik des Aufschubs nicht, zwar trifft und trifft keine Antwort-E-Mail ein - da jedoch als Figur in einem Roman ,mitzuspielen' prinzipiell anderen Gesetzen folgt als in einem Film als Schauspieler mitzuwirken, kann der schreibende Autor performativ erklären, dass Bruce Willis doch schon „längst drin“ ${ }^{\circ 6}$ in der Bank sei. Präsentisch (!) heißt es:

Aber ob Sie es nun wollen oder nicht: Sie sind in dieser Bank. Und ob Sie es nun wollen oder nicht: Die Bank ist umstellt. ${ }^{57}$

Wieder sind es die kleinen, recht unscheinbaren Wortarten, die nicht nur psychische Nähe kreieren, sondern - mehr noch - die schreibende Autor-Persona und ihren Adressaten in ein und denselben Verweisraum ${ }^{58}$ stellen, der in diesem Fall der gemeinsam betretene Textraum ist, und dabei ein absolutes Maximum an Proximität herstellen:

$D a$ ist eine hochschwangere Frau, Herr Willis, $d a$ ist ein alter Mann, der dringend seine Blutdruckmedikamente braucht, da ist dieser Kerl mit den großen Kopfhörern, der gerade mit sich aushandelt, ob er hier den Helden spielen soll, und wer weiß, wofür er sich entscheidet. ${ }^{59}$

Es sind Lokal- („da“, „hier“), Temporal- („gerade“) und Objektdeiktika („dieser“), die Autor-Persona und Figur in einen Verweisraum ziehen. Und tatsächlich betritt die Autor-Persona schließlich metaleptisch die eigene Geschichte, um sowohl in eigentlichem als auch in uneigentlichem Sinne im ,gleichen Boot‘ wie Bruce Willis auf der Flucht nach dem Banküberfall zu sitzen. Wieder kommen dabei Deiktika und auch das Präsens als Zeitform zu Hilfe, um ein gemeinsames hic et nunc ${ }^{60} \mathrm{zu}$ generieren: „Und jetzt sitze ich nicht am Schreibtisch, sondern hier mit Ihnen in diesem Boot." 61

55 Alle Teilzitate: Zeman 2016, 266.

56 Rammstedt 2012, 37.

57 Rammstedt 2012, 42.

58 Zum Beitrag von Deiktika zur Herstellung eines „Verweisraums“ vgl. Ehlich 2009, 69-70.

59 Rammstedt 2012, 42. [Herv. Y. M.]

60 Wiederholt wird ein „Hier und Jetzt“ heraufbeschworen, vgl. Rammstedt 2012, 66 u. ö.

61 Rammstedt 2012, 67. [Herv. Y. M.] 
Zwar erweist sich die ohne ihr Zutun in die Diegese hineingezogene BruceWillis-Figur als eher widerspenstig und unkooperativ, lässt ihren Autor kurz vor dem Ende im Stich und geht einfach, doch selbst noch in der Negation wird eine gemeinsame Geschichte erlebt. Den Schluss des Romans, die Rettung aus der „brenzligen Situation“, kann der von seiner Figur allein gelassene Autor trotzdem noch erzählen - nämlich einfach durch Voranstellung der Negationspartikel „nicht":

Sehr geehrter Herr Willis,

Sie wissen selbst, wie alles nicht gewesen ist. Sie wissen, dass Sie nicht nach ein paar Metern stehen geblieben sind. Sie wissen, dass Sie sich nicht noch einmal umgedreht haben, langsam und schwerfällig, als sei die Luft sehr zäh. Sie haben mich nicht angeblickt, nicht müde, nicht lustlos, nicht ganz und gar widerwillig. [...] $]^{62}$

Und so geht es über mehrere Seiten weiter. „Sie wissen“, erklärt die Autor-Persona, „dass es nicht so gewesen ist, Herr Willis, sonst müsste ich Ihnen das nicht schreiben. "63 Tatsächlich ist damit ein wesentlicher Schreibantrieb benannt. Während E-Mails wie diese in der physischen Kopräsenz eines für alle Beteiligten „unmittelbare[n], sinnlich zugängliche[n] Wahrnehmungsraum[s]"64 einer face to faceInteraktion nicht nur überflüssig, sondern schlichtweg unsinnig gewesen wären, schweißen sie Autor-Persona und Figur in einem „Textraum“ bzw. einem durch ihn eröffneten „Vorstellungsraum“ zusammen. ${ }^{65}$ Und genau das ist der Sinn des ganzen Schreibprojekts. In diesem Sinne kann man Rammstedts Roman als eine Huldigung an die Kraft der literarischen Imagination lesen. ${ }^{66}$

Was Rammstedts E-Mail-Roman in nahezu mustergültiger Manier zeigt, ist das Auseinanderklaffen von raumzeitlicher und psychischer Nähe. Die raumzeitliche Distanz ist nicht nur der Grund, weshalb überhaupt geschrieben werden muss, sondern dabei Bedingungs- und Erleichterungsfaktor der sprachlich konstruierten psychischen Nähe. Das allerdings ist weniger eine singuläre Einsicht denn seinerseits ein Topos der digitalen wie auch schon der analogen Briefliteratur. Radikalisiert findet sich diese Konstellation beispielsweise auch schon in dem im Jahr 2010 im russischen Original erschienenen, 2012 unter dem Titel Briefsteller ins Deutsche übersetzten Roman des in der Schweiz lebenden Schriftstellers Michail Schischkin, in dem die Briefpartner nicht nur zu unterschiedlichen Zeiten

62 Rammstedt 2012, 147.

63 Rammstedt 2012, 151.

64 Ehlich 2009, 70.

65 Vgl. Ehlich 2009, 70.

66 Metapoetisch auf den Punkt gebracht: „Als wir selbst sind wir alle verloren.“ (Rammstedt 2012, 30). 
leben, sondern Briefe selbst noch über den Tod hinaus ankommen. ${ }^{67}$ Es ließen sich problemlos weitere Beispiele für diesen Topos anführen, für das literarische Auseinanderklaffen von raumzeitlicher und psychischer Nähe in der digitalen wie in der analogen Briefliteratur, dies soll hier jedoch genügen.

Im Übrigen erhält die Autor-Persona in Rammstedts Roman am Ende doch noch eine Antwort-E-Mail. Diese aber liegt - in einem letzten metafiktionalen Clou - im paratextuellen Außenbereich, insofern sie schon nach der Danksagung am Buchende platziert ist. Es ist keine Nachricht von Bruce Willis, sondern eine E-Mail von ,Uma Thurman', die ihre Bereitschaft erklärt, zusammen mit anderen Hollywood-Schauspielern im „Buch mitzuspielen“, ihrerseits nun um „rasch[e]“ Antwort bittet und so ein konversationelles turn-taking einleitet. ${ }^{68}$

Das aber ist nicht mehr Gegenstand des Romans.

\section{Literatur}

Beebee, Thomas 0. (2006). Epistolary Fiction in Europe. 1500-1850. Cambridge.

Bürgel, Peter (1976). „Der Privatbrief. Entwurf eines heuristischen Modells“, in: Deutsche Vierteljahrsschrift für Literaturwissenschaft und Geistesgeschichte 50: 281-297.

Ehlich, Konrad (2009). „Deixis und Dichtung - Linguistische Überlegungen“, in: Gesprochen geschrieben - gedichtet. Variationen und Transformationen von Sprache. Hg. v. Monika Dannerer, Peter Mauser, Hannes Scheutz u. Andreas E. Weiss. Berlin: 67-79.

Ehlich, Konrad (1983). „Text und sprachliches Handeln. Die Entstehung von Texten aus dem Bedürfnis nach Überlieferung“, in: Schrift und Gedächtnis. Beiträge zur Archäologie der literarischen Kommunikation. Hg. v. Aleida Assmann, Jan Assmann u. Christof Hardmeier. München: 24-43.

Fürholzer, Katharina u. Yulia Mevissen (2017). „Einleitung: Briefkultur und Affektästhetik“, in: Briefkultur und Affektästhetik. Hg. v. dens. Heidelberg: 7-24.

Gellert, Christian Fürchtegott (1751). Briefe, nebst einer praktischen Abhandlung von dem guten Geschmacke in Briefen. Leipzig.

Glattauer, Daniel ( $\left.{ }^{40} 2008\right)$. Gut gegen Nordwind. München.

Hess-Lüttich, Ernest W. B. (1997). „E-Epistolographie: Briefkultur im Medienwandel“, in: Kultur -Medien - Macht. Cultural Studies und Medienanalyse. Hg. v. Andreas Repp u. Rainer Winter. Opladen: 225-246.

Koch, Peter u. Wulf Oesterreicher (1985). „Sprache der Nähe - Sprache der Distanz. Mündlichkeit und Schriftlichkeit im Spannungsfeld von Sprachtheorie und Sprachgeschichte“, in: Romanistisches Jahrbuch 36: 15-43.

67 Vgl. Schischkin 2012.

68 Rammstedt 2012, [unpag.]. 
La Roche, Sophie von (1771). Geschichte des Fräuleins von Sternheim. Von einer Freundin derselben aus Original-Papieren und andern zuverläßigen Quellen gezogen. Herausgegeben von C. M. Wieland. 2 Bde. Leipzig.

Lessing, Gotthold Ephraim (1967). Lessings Briefe in einem Band. Hg. v. d. Nationalen Forschungsund Gedenkstätten der klassischen deutschen Literatur in Weimar. Berlin u. Weimar.

Nickisch, Reinhard M. G. (1991). Brief. Stuttgart.

Oesterreicher, Wulf u. Peter Koch (2016). „30 Jahre ,Sprache der Nähe - Sprache der Distanz“. Zu Anfängen und Entwicklung von Konzepten im Feld von Mündlichkeit und Schriftlichkeit“, in: Zur Karriere von ,Nähe und Distanz: Rezeption und Diskussion des Koch-OesterreicherModells. Hg. v. Helmuth Feilke u. Mathilde Hennig. Berlin u. Boston: 11-72.

Rammstedt, Tilmann (2012). Die Abenteuer meines ehemaligen Bankberaters. Köln.

Schischkin, Michail (2012). Briefsteller. München.

Schlögl, Rudolf (2014). Anwesende und Abwesende. Grundriss für eine Gesellschaftsgeschichte der Frühen Neuzeit. Konstanz.

Seneca, Lucius Annaeus (1987). Philosophische Schriften. Lateinisch und Deutsch. 5 Bde. Hg. v. Manfred Rosenbach. Bd. 4: Ad Lucilium epistulae morales LXX-CXXIV, [CXXV]/An Lucilius Briefe über Ethik 70-124 [125] . Übers., eingeleitet u. mit Anmerkungen vers. v. Manfred Rosenbach. Darmstadt.

Siegert, Bernhard (1993). Relais. Geschicke der Literatur als Epoche der Post. 1751-1913. Berlin. Strobel, Jochen (2018). „Welchen Erkenntnisgewinn versprechen digitale Briefeditionen?“, in: Was ist ein Brief? Aufsätze zu epistolarer Theorie und Kultur / What is a letter? Essays on epistolary theory and culture. Hg. v. Marie Isabel Matthews-Schlinzig u. Caroline Socha. Würzburg: 91-105.

Stuck, Benjamin (2013). „Kulturphilosophie und Phänomenologie. Einige Ideen zum Begriff der Appräsentation bei Alfred Schütz und Edmund Husserl“, in: Der Begriff der Kultur. Kulturphilosophie als Aufgabe. Hg. v. Arbeitskreis Kultur- und Sozialphilosophie. Bielefeld: 25-45.

Ueding, Gert (2016). „Wechselrede. Rhetorische Anmerkungen zur europäischen Brieftheorie“, in: Cahiers d'Études Germaniques 70: 21-34.

Varatharajah, Senthuran (2016). Vor der Zunahme der Zeichen. Frankfurt a. M.

Vellusig, Robert (2011). „Aufklärung und Briefkultur. Wie das Herz sprechen lernt, wenn es zu schreiben beginnt“, in: Das achtzehnte Jahrhundert 35,2: 154-171.

Vellusig, Robert (2018). „Die Poesie des Briefes. Eine literaturanthropologische Skizze“, in: Was ist ein Brief? Aufsätze zu epistolarer Theorie und Kultur / What is a letter? Essays on epistolary theory and culture. Hg. v. Marie Isabel Matthews-Schlinzig u. Caroline Socha. Würzburg: 57-75.

Wegmann, Nikolaus (1988). Diskurse der Empfindsamkeit. Zur Geschichte eines Gefühls in der Literatur des 18. Jahrhunderts. Stuttgart.

Zaimoglu, Feridun (2002). Liebesmale, scharlachrot. Köln.

Zeman, Sonja (2016). „Nähe, Distanz und (Historische) Pragmatik. Oder: Wie ,nah“ ist ,Nähesprache'?“, in: Zur Karriere von ,Nähe und Distanz: Rezeption und Diskussion des KochOesterreicher-Modells. Hg. v. Helmuth Feilke u. Mathilde Hennig. Berlin u. Boston: 259-298. 
